\title{
State of the (net)work address Developing criteria for applying social networking to the work environment
}

\author{
André Calero Valdez ${ }^{\mathrm{a},}$, Anne Kathrin Schaar ${ }^{\mathrm{a}}$ and Martina Ziefle ${ }^{\mathrm{a}}$ \\ ${ }^{a}$ Human Computer Interaction Center, RWTH Aachen University, Theaterplatz 14, 52062 \\ Aachen, Germany
}

\begin{abstract}
In an increasingly faster paced innovative world, maintaining the ability to innovate in spite of an aging work force will become every company's strongest leverage for success. Tapping the latent knowledge resources and creativity of overlooked employees and persisting crucial information for business conduct are promising results for social networking sites (SNS) in a working context. Usability and usefulness are exponential factors in creating a successful SNS. In order to make a SNS usable for a heterogeneous user group, analyses of user diversity in regard to social media need to be done. Furthermore differences in communication medium and frequency in regard to age, content, hierarchy position, departmental thresholds and company size need to be analyzed. For analysis purposes both qualitative and quantitative research methods were applied. Strong effects of age and communication content were found in survey with 194 participants.
\end{abstract}

Keywords: social networks, user diversity, survey, SNS, collaboration, acceptance

\section{Introduction}

The feeling of connection is a core human need [14]. All major recent technological advancements, that redefined human living, improved human connectivity. The Internet connected people and information throughout the globe. The mobile phone allowed people to be connected everywhere and anytime. Being available and connected became an essential standard in the private and commercial sector [10]. These technologies arose from commercial requirements for an ever-increasing speed in information interchange and business processes. The personal need for connecting with your peers in the private sector, gave rise to highly successful social network technologies such as Myspace, Facebook, Twitter and others [9]. These allow people to be connected with and informed about the daily affairs of their friends independently of their whereabouts and time of day. New styles of communication became present and permeated massively into the work environment.
Globalization, opening of markets and offshore production shifted the focus of key business efforts in the leading industrial nations from production over to innovation and information exchange. Utilization of innovative and informative potential became critical factors for success, sustainability and competitive capacity of all major businesses [17]. This change is accompanied with fundamental changes in labor market abroad and employment biographies. Project work, short-term contracting, temporary employment, parental leave, retirement of baby boomers and the looming skills shortage require faster allocation of resources, conservation of business critical information and training of employees to ensure business competitiveness [4]. To face challenges like flexible working hours and international business relationships, supportive technologies will have to be implemented in the work environment.

The approach of social networking sites like Facebook can be facilitated to support communication and storage of knowledge within companies [11]. In or-

Corresponding author. E-mail: calero-valdez@humtec.rwth-aachen.de 
der to transfer the social network approach to the business context certain criteria have to be met in order to establish a flourishing community with active participants that benefit company innovation potential. Direct translation from public social networking sites to the work context might not be feasible, due to the specific character of the work context. Issues like privacy, control, functional scope and relationship topology possibly differ from those in private social networks in addition to different purposes of use [16].

\subsection{Usefulness of Social Networks}

Social networking sites depend heavily on user content and user participation, since they only provide computerized means of communication and information storage. In order to be useful for each individual user, SNSs need to attract all relevant users on a regular basis and persuade users into contributing to the network by sharing information and interacting with other users.

A SNSs' attractiveness to users should relate to the value of the network to the individual user. Metcalf's law [1] states that the value of a network is equal to the square of the number of connected users (i.e. the amount of all possible connections). Additionally Reed's law [12], which recognizes formation of subgroups within a network, even goes as far as to assume an exponential utility in relation to connected users. Although the human brains' capacity to handle relationships (i.e. approximately 150 connections) $[12,13]$ is probably the strongest limiting factor of a network's utility, each individual missing from a possible sub-network network limits the utility of the given network by a factor determined by both size of the network and value of the missing user.

Applying this to the working context, where the biggest possible user base is the total amount of employees plus the amount of possible customers, leads to the conclusion, that especially in small companies, every employee's participation is required for the network to flourish.

From the perspective of knowledge generation the SNS's value does not only depend on user's perceived utility of the network, but also on actual generation of knowledge both qualitatively and quantitatively. Larger networks decrease the level of commonality, reducing the level of collective intelligence to the level of the least common denominator of all users [15].
Allowing users to form sub-networks for special interests can counterbalance this. These sub-networks of like-mindedness increase the quality of communication within but are also detrimental to generation of new ideas, which benefit from having non-familiar ideas from group outsiders thus profiting from user heterogeneity.

In constructing a model of a SNS with different aspects of connectedness of users in mind, utility and attractiveness to the user can vary. It is important that the SNS is able to represent structures that relate to actual human connectedness (e.g. friendships) as well as work environment constraints (e.g. hierarchy) and knowledge generation beneficiary constructs (e.g. SIGs). All this needs to be done with a level of granularity that maximizes information interchange within and between groups but also SNS attractiveness and utility. This should allow a high level of motivation to use and contribute to the SNS.

\subsection{Paradigms of collaboration analysis}

The construction of a model used for creating a SNS strongly influences the SNSs capability to connect users, store information and enable creation of new knowledge. Different perspectives will lead to different interfaces, which will themselves lead to different workflows. Therefore it is important to keep in mind which paradigms are used for modeling analysis.

Resnyansky [15] stated three different paradigms by which collaboration technology can be analyzed.

The Network paradigm focuses on how users are connected with each other. Its key issues are Identity, Security and Trust. Users have a unique online identity, which is only usable by the rightful owner, which allows for a high degree of trust between connected users. This constitutes a strictly infrastructural view of social networking and does not focus on content (or medium).

The Communication paradigm focuses on the interactions that take place within a social network. Its key issue is tracking participants' contribution to the overall success of the collaboration. Users exchange documents, which they collaboratively compose. This medium centered approach leads to development of collaboration software that creates shared mutual change-awareness for its users.

The Production paradigm focuses on a transformation of the collaborative entity by analyzing users needs, concerns and behavior at the workplace. Its key issues are situational awareness, social order and 
heterogeneity synergy. It leads to creating a streamlined workflow as a form of allowing users to express themselves in regard to their needs and concerns within a given situational context. This shaping of workflow allows participation of non-experts and thus benefits by leveraging synergetic effects of user heterogeneity. At the same time it allows management by the social order of recognized individual competence such as experts or organizational hierarchy. This approach includes both medium and infrastructure.

In order to create successful SNS for the workplace all aspects of the three different paradigms need to be considered. A thorough analysis of organizational communication structures, contend and medium of communication, and users needs is important for planning a future SNS.

\subsection{Social network as an active participant}

In a successful application of a SNS the used platform is not only a means of infrastructure or workflow streamlining, but also an active participant in the knowledge creation process [15]. It supports the users by not only allowing sharing of digital but also conceptual resources. It allows collaboration of heterogeneous user groups with asymmetrical relationships, needs and wants as well as different levels of expert knowledge. The SNS needs to convey an emotional translation of knowledge by collaborative knowing to ensure high acceptance on behalf of the users.

\section{Method}

In order to create a suitable social networking site for the work environment it is essential to include the users into the development process. Initially two aspects need further investigation to guarantee a successful rollout:

1. Usage of Social Media in the workforce

2. Communication structures in business contexts

To ensure a broad and deep insight into these aspects, both qualitative and quantitative methods were applied. At first focus groups were conducted with a total ten participants to generate preliminary results for the construction of a follow-up questionnaire. Main aspects of the focus group were: (1) How do differences between age groups, gender, technical expertise, social media adaption regard to social net- working. (2) How does user diversity regard to differences in business conduct, hierarchy, communication formality, communication medium and flow of information.

In a second step the results of the focus groups were used to create a questionnaire to verify the findings quantitatively. Fifty participants were acquired to take part in this study. As independent variables demographic data was chosen (i.e. age, gender) as well as technical expertise and social media adoption. These were measured in a six-point-Likert scale and then assigned to two different groups (high vs. low expertise/level of adoption). As dependent variables (1) Perceived Ease of Use/Usefulness, usage context/frequency of different social networks were assessed, as well as (2) communication frequency, medium, content, hierarchy and departmental transgression were assessed.

\subsection{Focus Groups}

In order to explore hypotheses about social networking effects on communication focus groups were conducted prior to the questionnaire survey. Based on the qualitative results from this focus group hypotheses and survey questions were designed.

\subsubsection{Focus group setup}

Two focus groups were conducted. In both focus groups participants were addressed through social networks of students and researchers participating in the focus group. The participants were chosen with a focus on age diversity and IT-based work was chosen in order to obtain helpful input for further research. The focus groups each took about 90 minutes focused users' social media adaption, technical expertise and how the participants used IT in their everyday lives and at work. Additionally questions regarding feature wishes for a future social networks in the work environment were asked along with perceived benefits or risks of this features.

The focus group participants were in both cases a group of two students (mid twenties, male and female), two younger members of the workforce (in their 30ies, male and female) and an older member of the workforce (mid-fifties, male).

\subsubsection{Results of the focus group}

The focus groups revealed that users differ strongly in their usage patterns of social networks. Being a heavy user of one social network, does not translate to being a general heavy user of social networks. All 
participants who work in IT related jobs have used both social networks and knowledge management systems in their jobs and had reported a rather mixed acceptance of the used technology. While private usage of SNS like Facebook was widely accepted, similar concepts at work were mostly rejected.

A picture of a few heavy contributors during launch-phase and lots of passive consumers during the first few weeks of the SNS leading to fewer and fewer active participation and a lot of unmaintained pages emerged. Only if the company enforced a strong policy usage was stable but nonetheless considered cumbersome. Participants who worked for bigger companies reported higher acceptance of SNS.

Only one participant reported a high acceptance of a Wiki-System. It is in use at her students' union executive committee in which she participated without pay. This supports the hypothesis that voluntariness of use $[2,5]$ strongly effects acceptance of SNS.

Another aspect that emerged is that certain types of media (chat, email, phone) were only used in certain situational contexts. Usage barriers were mostly the feeling of being observed, fear of control of private communication and bad usability and workflow integration. Features requests like active support during workflow were also mentioned.

\subsection{Questionnaire}

The questionnaire study was conducted with an online survey system (surveymonkey.com). The online questionnaire was sent through email to both a preselected target audience (companies with IT background, software engineering, university departments, banking) as well as open questionnaire posted to social media related forums/twitter (reply rates were equal +-5 ). Time required to answer the complete questionnaire was on average 15 minutes. The questionnaire was designed in order to answer the questions that came up during the focus groups.

\subsubsection{Independent variables}

As independent variables different demographic data were collected. Participants gender, age, education were assessed as well as private social media usage and behavior. Typical SNS were used, that are familiar to a German audience (Facebook, meinVZ, myspace, Twitter). For these SNS usage frequency was assessed with a five-point-Likert scale (UsageFrequency (UF) Scale: $1=$ daily, $2=$ several times a week, 3 =several times a month, $4=$ rarer, $5=$ never).
For deeper insights, the amount of relationships on the favorite SNS was also measured.

In order to keep the methodology similar to earlier research $[3,7,8]$ perceived locus of control over technology was assessed using eight questions on a six-point-Likert scale (range: 8-48, Cronbach's Al$\mathrm{pha}=.91$ ). Additionally several key factors regarding the participants employer were asked (company size, position within company).

\subsubsection{Dependent variables}

As dependent variables different communications behaviors and media were assessed. It was asked what type of medium (vis-á-vis/face to face, phone, mobile phone, email, text-messaging, instant messenger, SNS) was used in what circumstances (private / business, with customers, inter- / intradepartmental and cross-hierarchy) and how often they were used on a five-point-Likert scale (see above for UF scale).

\subsubsection{Participants}

The participants for the questionnaire were reached by sending email-invitations to previously selected possible participants. Most of them were chosen for their work background. The URL for the online-survey was posted on social networks additionally. In the questionnaire study 194 participants took part, of which 97 were male and 97 were female.

The average age (see Figure 1) of the participants was 31.9 years $(\mathrm{SD}=9.9$, range $=17-66$ years $)$ with males being slightly older $\left(\mathrm{M}_{\text {male }}=33.2 \mathrm{SD}_{\text {male }}=10.5\right)$ than females $\left(\mathrm{M}_{\text {female }}=30.7 \quad \mathrm{SD}_{\text {female }}=9.5\right)$. Age showed a bimodal distribution, probably due to the way of acquiring participants through personal networks of the involved researchers (peers and parents).

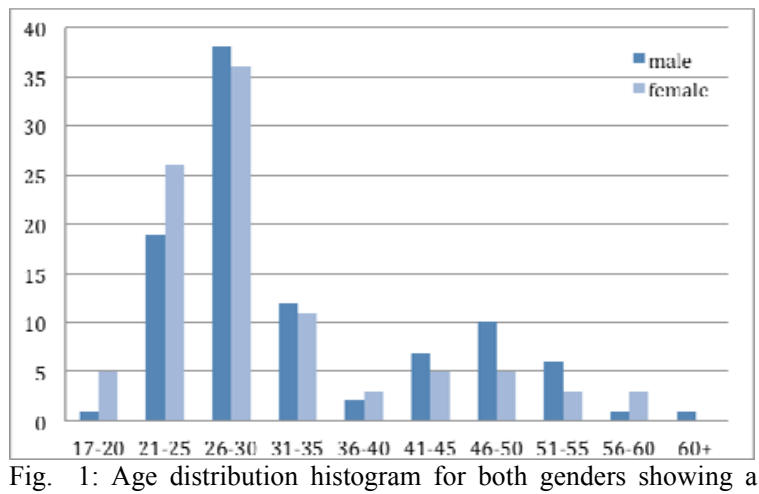
slightly bimodal distribution.

The biggest group of participants had already finished their university education $(45.9 \%)$ or even fin- 
ished a doctoral degree (1.5\%). A large majority of the participants finished their school education $(38.7 \%)$ but the smallest amount of participants were either industrially trained $(10.8 \%)$ or still in their school education (3.1\%). No significant effect of gender on education was found in this sample $(t(194)=0.48 \mathrm{p}=.63)$. Correlation analysis shows that age is correlated with a higher position in the company $(\mathrm{r}=.39, \mathrm{p}<.01)$ and also company size $(\mathrm{r}=.17$, $\mathrm{p}<.01$ ), which is expected, since people climb the hierarchy as they get older.

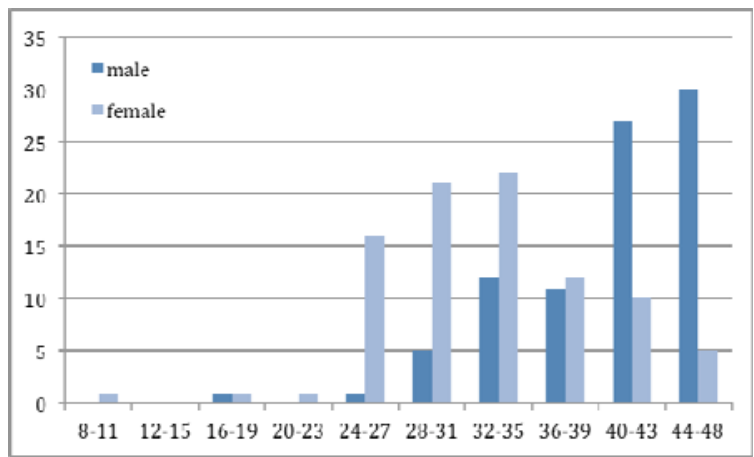

Fig. 2: Male and female KUT scores histogram.

When looking at the participants self reported locus of control over technology (KUT) males $(\mathrm{M}=40.08, \quad \mathrm{SE}=0.62)$ reported significantly higher feeling of control than females $(\mathrm{M}=33.04, \mathrm{SE}=0.72)$. $t(174)=7.38, \mathrm{p}<.001, \mathrm{r}=.49$ (see Figure 2). Age and KUT did not correlate $(\mathrm{r}=-.03)$.

\section{Results}

The results were analyzed using $t$-Tests, one way ANOVAs, bivariate correlations and multivariate analysis of variance with a level of significance set at $5 \%$. For correlational analysis Spearman's rho was used.

\subsection{Social media behavior}

In regard to social media adoption Facebook was the social networking site that was used most frequently in comparison to meinVZ, myspace and Twitter $\left(\mathrm{M}_{\text {Facebook }}=2.37 \mathrm{SD}_{\text {Facebook }}=1.72, \mathrm{M}_{\text {meinVZ }}=4.21\right.$ $\mathrm{SD}_{\text {meinVZ }}=1.15, \mathrm{M}_{\text {myspace }}=4.91 \quad \mathrm{SD}_{\text {myspace }}=.59, \mathrm{M}_{\text {Twit- }}$ ter $=4.79 \mathrm{SD}_{\text {Twitter }}=0.82$ ). It was also only $\mathrm{SNS}$ that showed a difference in mean of usage frequency between genders. Males use Facebook less frequently $(\mathrm{M}=2.69 \mathrm{SE}=0.02)$ than females $(\mathrm{M}=2.06 \mathrm{SE}=0.17)$, $t(187)=2.54, \mathrm{p}<.05, \mathrm{r}=.18$. But the effect is too small for the sample size to be considered a genuine effect. Age correlates negatively with usage frequency of Facebook $(\mathrm{r}=.68, \mathrm{p}<.01)$, showing that social media are predominately used by younger adults.

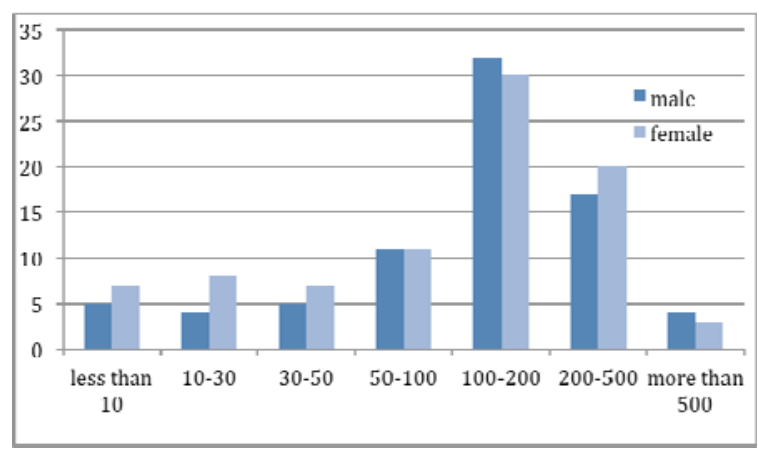

Fig. 3: Number of "Friendship" relationships on the user's favored SNS in regard to gender.

The amount of people that you are connected with on a SNS is equal for both sexes $(\mathrm{t}(170)=0.74 \mathrm{p}=.46$, see Figure 3). Something that differed between genders is the proportion of which the users interacts with both online and offline on a regular basis. Males mostly report that that they talk to only $10 \%$ of their connected users both offline and online, while females report that they interact with approximately $25 \%$ of their connected friends (see Figure 4, onlineinteraction: $t(168)=-3.05 \mathrm{p}<.01$, offline-interaction: $t(170)=-2.10 \mathrm{p}<.05)$.

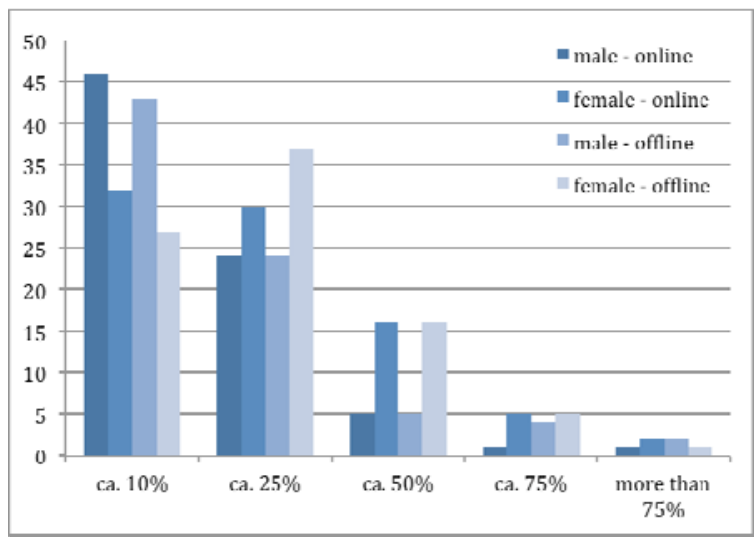

Fig. 4: Histogram of relative amount in regard to total connections of actual interaction both online and offline for both genders.

Correlation analysis shows that the amount of relationships correlates negatively with age $(\mathrm{r}=-.58$ $\mathrm{p}<.01$ ), positively with a high feeling of control over technology $(\mathrm{r}=.19, \mathrm{p}<.05)$, highly with usage frequency of Facebook $(\mathrm{r}=-.61, \mathrm{p}<.01)$ and highly with 
the people that a user actually interacts with online $(\mathrm{r}=-.25, \mathrm{p}<.01)$.

\subsection{Workspace communication}

Different media are used differently by both genders in different contexts. Seven media were surveyed: Vis-á-vis communication, calling on a landline phone, calling on a mobile phone, texting on a mobile phone, sending email, using an instant messenger and using a SNS for communication. Ten different scenarios were assessed in the survey: Communication with...

1. Customers

2. Colleagues that you don't consider your friends about business topics

3. Colleagues that are your friends about business topics

4. Colleagues that are your friends about private topics

5. Friends about private topics at the workplace

6. Members of your department

7. Members of other departments

8. Subordinates

9. Superiors

10. Customers (how the participant thinks everybody else communicates with customers).

They were ranked on a five-point-Likert scale (see above for UF-Scale). Plotting theses values shows, that communication mostly differs when you have friendly relationships or personal matters to discuss.

Figure 5 shows that business related communication mostly relies on email, phone or vis-á-vis communication. Vis-á-vis communication is especially important in intra-departmental communication.

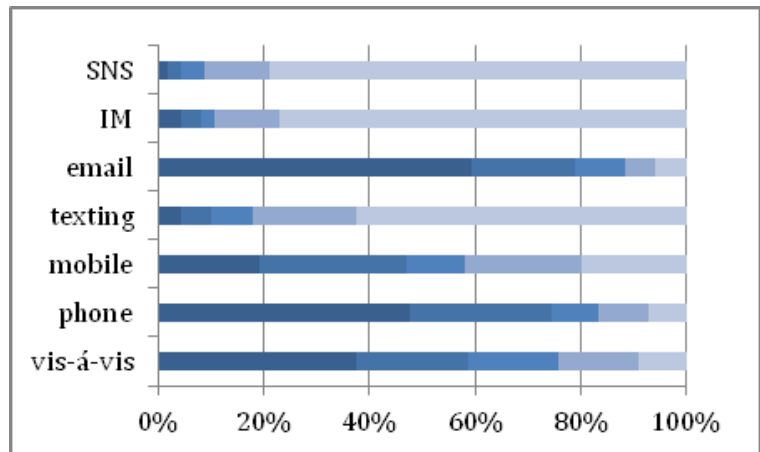

Fig. 5: Scenario 1 - Communication with customers (darker shade means heavier use of this medium). This graph is highly similar to those graphs of scenarios 2, 6 (heavier use of vis-á-vis), 7, 8, 9, 10.

As soon as friendly relationships are part of the communication other media are also used more heav- ily. Especially SNS, IM, texting become more important (see Figure 6) independently of the content of the conversation (business or private matters).

Correlation analysis shows (see Appendix for correlation tables) that age, company size and position in hierarchy have an impact on what medium is used for communication. The older a participant is the less likely will he use SNS in all but one scenario (see Table 1, r >0).

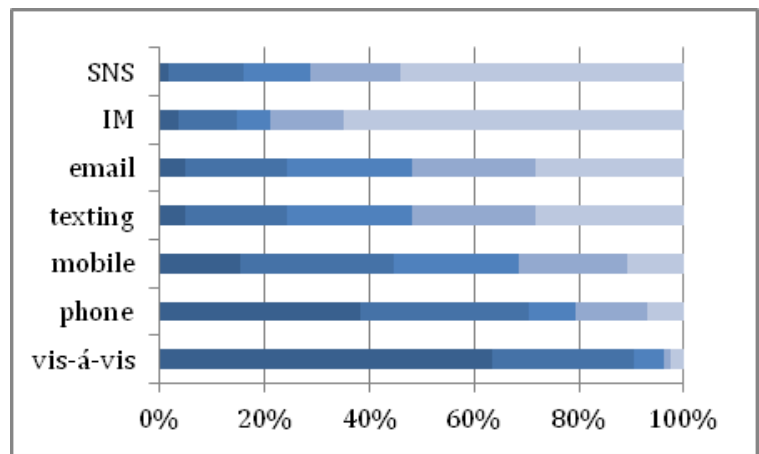

Fig. 6: Scenario 3 - Communication with colleagues that are you friends (darker shade means heavier use of this medium). This graph is highly similar to those graphs of scenario 4, 5 (lighter use of vis-á-vis).

Especially using mobile phones, texting, emailing or using an instant messenger is less often used by older users in private contexts. If the company is bigger users will be less likely to participate in communication through texting, IM and SNS, and have less communication about private topics.

Interestingly KUT showed no correlation with any usage frequency of all media in any context. This was not expected as the level of technical selfconfidence is well documented as a major facilitator and obstructer, respectively, on all kinds of technology handling $[6,18]$.

\section{Discussion}

Differences in usage behavior are essential to be incorporated into designing a SNS for the work environment. Also, user preferences regarding their favorite electronic media Preferred media should be included into different workflows to enable a seamless non-obtrusive workflow. Since they appear frequently in all workflows especially email and phone need an easy to reach implementation, like a "call now" function included on a persons profile page, which connects to a companies phone system and automatically places a call. 
Older users are rather unfamiliar with using social networking sites, Instant Messengers and texting on mobile phones. Therefore these functions should be looked into more deeply to understand why older users refrain from using them. If they turn out to be very useful from a perspective of younger users to their workflow (e.g. asynchronicity of instant messengers), interface redesign or integration into other media workflows should be considered to make them more usable to older users. Surprisingly KUT showed no influence on media usage in any context. On the base of the present data we cannot explain the missing link in this study. The use of social media could possibly be driven by different using motives in comparison to conventional ICT technology. One could speculate that using motives of social networks are more influenced by social needs and communication wishes that are stronger than the perception of using a technology. Further research is required.

Formality of communication increases as the companies become larger. This is especially true, when communication happens between subordinates and superiors. A SNS should allow formalized communication in order to incorporate the need of formality between departments and hierarchical borders.

Dunbar's number seems to reflect the average size of people's networks on their favorite SNS, although they actively interact with only between $10-25 \%$ of this number. The biggest influence on the number of relationships was the participant's age but also KUT showed an influence on amount of relationships.

The results in general show that it is useful to respect user differences in development of social networks, because heterogeneous users demand a customizable community to assure successful adoption throughout different work environment. Social structures such as SIGs, work hierarchy and friendship relationships need to find their counterpart within a SNS to allow natural forming of groups and a fitting usage of communication medium for their specific content.

\subsection{Limitations of this study}

This study was exploratory in nature, which is visible in the non-normal properties of the sample histogram. In order to ensure all found effects are considerably large enough, more people need to be surveyed $(>750)$. They should especially fill the age gap between 30 and 45 year olds in order to restore normality and resemblance to the whole population. Further research is required to ensure which effects are interconnected and to create a model of prediction of which user aspects affect what communication medium. Only the strongest effects were reported.

\section{References}

[1] "Metcalfe's Law", Wikipedia.org 2011; http://en.wikipedia.org/wiki/Metcalfe's_law

[2] R. Agarwal, and J. Prasad, (1997), The Role of Innovation Characteristics and Perceived Voluntariness in the Acceptance of Information Technologies. Decision Sciences, 28: 557-582.

[3] F. Alagöz et al. (2010). From Cloud Computing to Mobile Internet, From User Focus to Culture and Hedonism: The Crucible of Mobile Health Care and Wellness Applications. IEEE 5th International Conference on Pervasive Computing and Applications (pp.38-45).

[4] J. Allmendinger, C. Ebner, 2006, Arbeitsmarkt und demographischer Wandel - Die Zukunft der Beschäftigung in Deutschland, Zeitschrift für Arbeits- und Organisationspsychologie 50 \#4, 227-239, Hogrefe Verlag, Göttingen

[5] J.E. Anderson, P.H. and Schwager, "SME Adoption of Wireless LAN Technology: Applying the UTAUT Model" (2004). SAIS 2004 Proceedings. Paper

6. http://aisel.aisnet.org/sais2004/6

[6] K. Arning, M. Ziefle, Different Perspectives on Technology Acceptance: The Role of Technology Type and Age, in: A Holzinger, K. Miesenberger (eds.), Human-Computer Interaction for eInclusion, LNCS 5889, Springer, 2009, pp. 20-41.

[7] G. Beier, (1999). Locus of control when interacting with technology [Kontrollüberzeugungen im Umgang mit Technik]. Report Psychologie, 24, 684-693.

[8] A. Calero Valdez, et al. (2010). Mental Models of Menu Structures in Diabetes Assistants. In: K. Miesenberger et al. (Eds.): 12th ICCHP- International Conference on Computers helping people with special needs. Part II, LNCS 6180 (pp. 584-591) Berlin, Heidelberg: Springer

[9] D. M. Boyd, N. B. Ellison, 2008, Social Network Sites: Definition, History, and Scholarship. Journal of ComputerMediated Communication, 13: 210-230.

[10]M. Castells, 2000, The Rise of the Network Society, Malden, Massachusetts, Blackwell.

[11] T.H. Davenport, L. Prusak, 2000, Working Knowledge - How organizations manage what they know, Harvard Business School Press.

[12]R. Dunbar, 1998, "Grooming, gossip, and the evolution of language", Harvard Univsity Press

[13]R. Dunbar, 1993, "Coevoluion of neocortical size, group size and language in humans, Behavioral and Brain Sciences 16 (4): 681-735

[14]A. H. Maslow, 1954, Motivation and Personality, Harper \& Row, New York.

[15]L. Resnyansky, 2011, "Global ICT-Mediated Collaborations: Thinking Beyond Networks", Technology and Society, IEEE, Vol 30, Issue 2: pp. 22-30

[16]A. Richter, M. Koch, 2008, Functions of Social Networking Services, COOP 08, Parina Hassanaly et al. (ed.), 87-98.

[17] T. J. Sturgeon, 1997, Does Manufacturing Still Matter? The Organizational Delinking of Production from Innovation, Roundtable on the International Economy, UC Berkeley.

[18] W. Wilkowska, M. Ziefle (2011). User diversity as a challenge for the integration of medical technology into future home environments, in: M. Ziefle and C. Röcker (eds.), Human-Centred Design of eHealth Technologies. Concepts, Methods and Applications. Hershey: P.A. IGI Global, 2011 
[19]M. Ziefle, A.K. Schaar, (2010). Technical Expertise and its Influence on the Acceptance of Future Medical Technologies.

What is influencing what to which extent? In G. Leitner, M.

Hitz \& Andreas Holzinger (eds). HCI in Work \& Learning,

Life \& Leisure, Lecture Notes in Computer Science 6389 (pp.

82-100). Berlin, Heidelberg: Springer.

Appendix.

Table 1: Correlation table for age with different media in all scenarios. * is sig. (two-tailored) at level $0.05, * *$ at level 0.01 .

\begin{tabular}{cccccccc} 
Scenario & Vis-á-vis & Phone & Mobile & Texting & Email & IM & SNS \\
\hline 1 & $-.193^{*}$ & $-.180^{*}$ & -0.061 & -0.018 & 0.010 & 0.099 & $.165^{*}$ \\
\hline 2 & -.065 & .056 & .043 & .102 & .124 & .102 & $.195^{*}$ \\
\hline 3 & -.087 & -.050 & .118 & $.260^{* *}$ & .105 & $.239^{* *}$ & $.371^{* *}$ \\
\hline 4 & .148 & .126 & $.209^{* *}$ & $.330^{* *}$ & $.291^{* *}$ & $.244^{* *}$ & $.408^{* *}$ \\
\hline 5 & .053 & .120 & $.218^{* *}$ & $.369^{* *}$ & $.263^{* *}$ & $.298^{* *}$ & $.440^{* *}$ \\
\hline 6 & .000 & .065 & .075 & .148 & $.214^{* *}$ & $.190^{*}$ & $.192^{*}$ \\
\hline 7 & -.053 & -.015 & -.004 & .052 & .059 & .148 & $.162^{*}$ \\
\hline 8 & $.163^{*}$ & .077 & .051 & .081 & $.227^{* *}$ & $.156^{*}$ & $.173^{*}$ \\
\hline 9 & $-.214^{* *}$ & -0.047 & -0.031 & 0.051 & -0.061 & $.163^{*}$ & $.159^{*}$ \\
\hline 10 & -.020 & .016 & .054 & .075 & .061 & $.178^{*}$ & .102
\end{tabular}

Table 2: Correlation table for "company size" with different media in all scenarios. * is sig. (two-tailored) at level $0.05, * *$ at level 0.01 .

\begin{tabular}{|c|c|c|c|c|c|c|c|}
\hline Scenario & Vis-á-vis & Phone & Mobile & Texting & Email & IM & SNS \\
\hline 1 & .104 & .072 & $.178 *$ & $.237 * *$ & .007 & $.215^{* *}$ & $.299 * *$ \\
\hline 2 & .016 & -.070 & .147 & $.281 * *$ & -.112 & $.207 * *$ & $.333 * *$ \\
\hline 3 & .047 & -.003 & .146 & $.184^{*}$ & .002 & .131 & $.250^{* *}$ \\
\hline 4 & .129 & $.239 * *$ & $.208^{* *}$ & $.292 * *$ & $.282^{* *}$ & $.187 *$ & $.314 * *$ \\
\hline 5 & $.224 * *$ & $.352 * *$ & $.256^{* *}$ & $.342 * *$ & $.334 * *$ & $.208^{* *}$ & $.239 * *$ \\
\hline 6 & .086 & -.130 & .147 & $.248 * *$ & -.037 & $.227 * *$ & $.332 * *$ \\
\hline 7 & .062 & -.043 & .139 & $.312 * *$ & -.066 & $.271^{* *}$ & $.328 * *$ \\
\hline 8 & .092 & .014 & .075 & $.261 * *$ & -.007 & $.280 * *$ & $.331 * *$ \\
\hline 9 & $.205^{* *}$ & $.194 *$ & $.213 * *$ & $.330 * *$ & .148 & $.377 * *$ & $.400 * *$ \\
\hline 10 & $.156^{*}$ & .130 & $.159 *$ & $.178^{*}$ & .124 & $.206^{* *}$ & $.245^{* *}$ \\
\hline
\end{tabular}


A.C. Valdez et al. / State of the (Net)work Address

Table 3: Correlation table for "hierarchy position" with different media in all scenarios. * is sig. (two-tailored) at level 0.05, ** at level 0.01 .

Scenario

Vis-á-vis

\begin{tabular}{cccccccc}
\hline 1 & -.010 & .003 & .041 & $.276^{* *}$ & .081 & $.251^{* *}$ \\
\hline 2 & .120 & $.206^{* *}$ & .128 & $.235^{* *}$ & $.155^{*}$ & $.237^{* *}$ \\
\hline 3 & .063 & .120 & .133 & $.297^{* *}$ & .060 & $.242^{* *}$ \\
\hline 4 & $.159^{*}$ & $.189^{*}$ & $.158^{*}$ & $.254^{* *}$ & $.207^{* *}$ & $.221^{* *}$ \\
\hline 5 & $.184^{*}$ & $.187^{*}$ & $.274^{* *}$ & $.316^{* *}$ & $.264^{* *}$ & $.253^{* *}$ \\
\hline 6 & $.171^{*}$ & $.294^{* *}$ & $.174^{*}$ & $.302^{* *}$ & $.244^{* *}$ & $.258^{* *}$ \\
\hline 7 & .121 & $.266^{* *}$ & $.184^{* *}$ & $.328^{* *}$ & $.266^{* *}$ & $.308^{* *}$ \\
\hline 8 & $.388^{* *}$ & $.279^{* *}$ & $.174^{*}$ & $.327^{* *}$ & $.454^{* *}$ & $.344^{* *}$ \\
\hline 9 & $-.155^{*}$ & -.065 & -.034 & .119 & -.069 & $.345^{* *}$ \\
\hline 10 & .020 & .021 & .039 & $.253^{* *}$ & -.015 & $.218^{* *}$ \\
\hline
\end{tabular}

